\title{
Antimycotic activity of Myrtus communis $L$. towards Candida spp. from clinical isolates
}

\author{
Sara Cannas ${ }^{1}$, Paola Molicotti ${ }^{1}$, Melania Ruggeri ${ }^{1}$, Marina Cubeddu ${ }^{1}$, Maurizio Sanguinetti ${ }^{2}$, Bruno \\ Marongiu $^{3}$, Stefania Zanetti ${ }^{1}$ \\ ${ }^{1}$ Dipartimento di Scienze Biomediche, Microbiologia Sperimentale e Clinica - Università degli Studi di Sassari, Italy \\ ${ }^{2}$ Istituto di Microbiologia, Università Cattolica del Sacro Cuore, Largo F. Vito 1, 00168 Rome, Italy \\ ${ }^{3}$ Dipartimento di Scienze Chimiche, Cittadella Universitaria di Monserrato, 09042 Monserrato, Italy
}

Key words: Myrtus communis; Candida spp.; essential oil

J Infect Dev Ctries 2013; 7(3):295-298.

(Received 19 June 2012- Accepted 16 November 2012)

Copyright (C) 2013 Cannas et al. This is an open-access article distributed under the Creative Commons Attribution License, which permits unrestricted use, distribution, and reproduction in any medium, provided the original work is properly cited.

\section{Introduction}

Myrtle (Myrtus communis L.) is an evergreen shrub belonging to the family of Myrtaceae, which grows spontaneously throughout the Mediterranean area. In Italy it grows along the coasts and on the internal hills, and it is abundant especially in the islands, where it is one of the most characteristic species. The essential oil obtained from its leaves and sometimes from its flowers and berries has been used for its tonic and medicinal properties, and it is used in the flavour and fragrance industries. Essential oils are gaining remarkable interest for their potential multipurpose use as antioxidant, antibacterial and antiseptic agents [1]. Myrtle berries and leaves are mostly employed for the industrial formulation of sweet liquors with digestive properties; however, the essential oil obtained from myrtle leaves has been used in the treatment of lung disorders, and Myrtus communis has a history in popular and traditional medicine [2]. Myrtus communis has exhibited the biological activities of tannins including anticancer and antioxidant activities [3]. In previous studies, we obtained encouraging results when we evaluated the antimicrobial properties of myrtle essential oil against several clinical strains, in particular against Helicobacter pylori and Mycobacteria [4,5].

Candidemia is the fourth most common nosocomial bloodstream infection. The incidence of candidemia has risen worldwide over the last two decades in different settings, mostly due to an increase in the use of more aggressive therapy practices, especially intensive chemotherapy for hematological malignancies, transplantation, and intensive care unit (ICU) use. To a lesser extent, the use of immunosuppressive agents for the treatment of autoimmune and other diseases, and even for the prolongation of life, has created a previously nonexistent immunocompromised population. Candida albicans is the most causative agent involved with fungal infections [6,7], and Candida non albicans are known to be even more resistant to conventional antifungal therapy. Candidemia is associated with considerable mortality. The management of Candida infections faces many problems, such as a limited number of antifungal drugs, toxicity, resistance of Candida to commonly used antifungal drugs, relapse of Candida infections, and the high cost of antifungal drugs $[8,12]$. It is therefore essential to discover new antifungal agents to combat strains expressing resistance to the available antifungal drugs. Natural products have been used as therapeutic agents and about half of the drugs that we use today are derived from natural sources [2,13]. Because of the development of microbial resistance, natural products are a valuable source of new antibiotic drugs [2]. Aromatic plants have been used since ancient times for their medicinal properties. Essential oils (EO) are complex mixtures of volatile compounds that result from secondary metabolic pathways of plants $[13,14]$.

The aim of this work was to evaluate the antimycotic activity of myrtle's essential oil toward five species of yeasts isolated from clinical samples (blood cultures): C. albicans (10 strains), C. glabrata (10 strains), C. krusei (10 strains), C. tropicalis (10 
Table 1. In vitro susceptibility of Candida spp. isolates to myrtle oil and 6 antifungal drugs

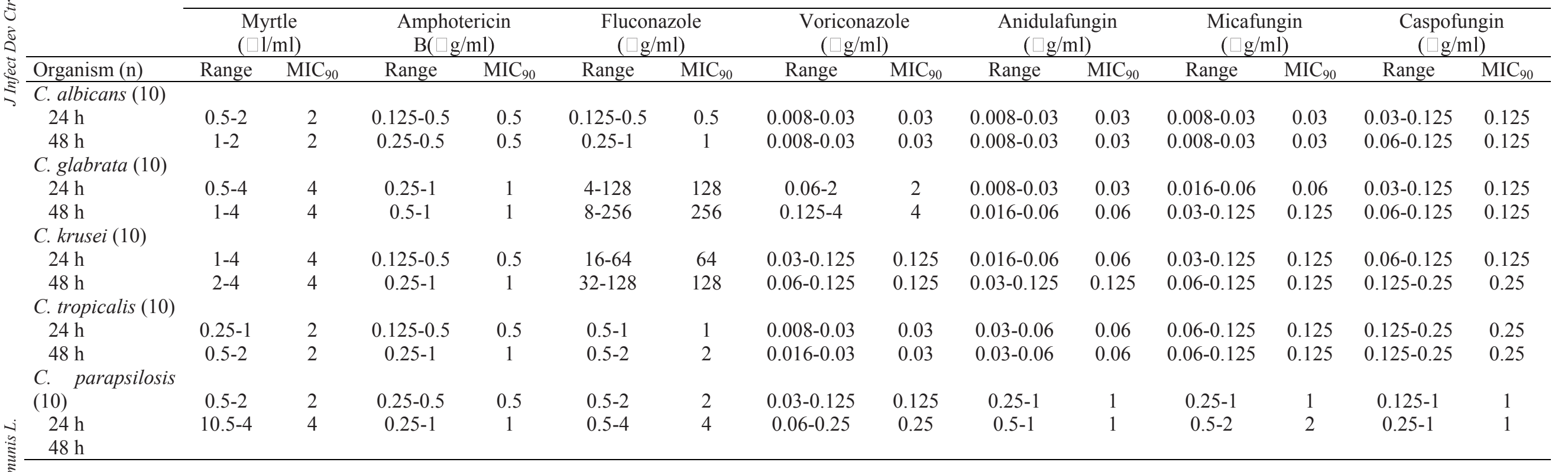


strains), and C. parapsilosis (10 strains). The results obtained at 24 and 48 hours were compared with the MIC using the following six antimycotics: amphotericin B, fluconazole, voriconazole, anidulafungin, micafungin, and caspofungin. The results obtained showed good antimycotic activity of myrtle's essential oil toward the five yeasts at the 24and the 48-hour time points.

\section{The study}

In this study the isolation of essential oils from Myrtus communis leaves was obtained by a hydrodistillation method using a Clevenger-type apparatus, according to the Italian Official Pharmacopoeia. The composition of the essential oils was analysed by GC/MS. A collection of 50 isolates belonging to five different species of Candida spp. was selected for this study: C. albicans (10), C. glabrata (10), C. krusei (10), C. tropicalis (10), and $C$. parapsilosis (10). The isolates were cultured from blood samples of hospitalized patients at the Institute of Microbiology Policlinico A. Gemelli in Rome. All microorganisms were identified by standard methods (germe tube test) and stored on Sabouraud dextrose agar plates until the study was performed [15]. The minimum inhibitory concentration (MIC) of myrtle oil was determined using the M27-A3 method [16], appropriately modified. Yeasts were cultivated at $37^{\circ} \mathrm{C}$ on Sabouraud agar plates (Kima, Padova, Italy) for 24 hours. The inoculum was prepared by a dilution of the colonies in salt solution, at a 0.5 McFarland concentration, and the concentration was confirmed by a spectrophotometric reading at a wavelength of 530 $\mathrm{nm}$. The sensitivity test was performed in RPMI 1640, using 96-well plates. Myrtle oil concentrations were prepared by serial one to two dilutions from $32 \mu \mathrm{g} / \mathrm{ml}$ to $0.06 \mu \mathrm{g} / \mathrm{ml}$. After shaking, $100 \mu \mathrm{l}$ of each oil dilution and $100 \mu \mathrm{l}$ of yeast suspension were added to each well and then incubated at $35^{\circ} \mathrm{C}$ for 48 hours. MIC was defined as the lowest concentration able to inhibit the fungal growth of $50 \%$ compared to the growth control and MIC values registered at 24 and 48 hours. Each isolate was tested in duplicate. Furthermore, each yeast strain included in the study was tested for its sensitivity to amphotericin B, fluconazole, voriconazole, caspofungin, anidulafungin, and micafungin following the M27-A3 protocol and using the CLSI breakpoints to establish susceptibility/resistance of the studied isolates [16]. Quality control strains (Candida parapsilosis ATCC 22019 and Candida krusei ATCC 6258) were incorporated in each set of experiments.

\section{Results}

The MIC data for the Candida spp. are summarized in the Table. After 24 hours the ranges of MIC obtained with myrtle essential oil were 0.5-2 $\mu \mathrm{g} / \mathrm{ml}, 0.5-4 \mu \mathrm{g} / \mathrm{ml}, 1-4 \mu \mathrm{g} / \mathrm{ml}, 0.25-1 \mu \mathrm{g} / \mathrm{ml}$, and $0.5-2$ $\mu \mathrm{g} / \mathrm{ml}$ for C. albicans, C. glabrata, C. krusei, C. tropicalis and $C$. parapsilosis respectively; MIC90 were $2 \mu \mathrm{g} / \mathrm{ml}$ for $C$. albicans, $C$. tropicalis and $C$. parapsilosis, while MIC90 for $C$. glabrata and $C$. $k r u s e i$ were $4 \mu \mathrm{g} / \mathrm{ml}$. After 48 hours the ranges of MIC were 1-2 $\mu \mathrm{g} / \mathrm{ml}$ for $C$. albicans, 1-4 for C. glabrata, 24 for $C$. krusei, $0.5-2 \mu \mathrm{g} / \mathrm{ml}$ for C. tropicalis and 0.5-4 $\mu \mathrm{g} / \mathrm{ml}$ for C. parapsilosis. MIC90 were $2 \mu \mathrm{g} / \mathrm{ml}$ for $C$. albicans, C. tropicalis, and $4 \mu \mathrm{g} / \mathrm{ml}$ for C. glabrata, $C$. krusei and C. parapsilosis. Furthermore, C. glabrata and $C$. krusei were resistant to fluconazole (MIC90: 128 and $64 \mu \mathrm{g} / \mathrm{ml}$ respectively after 24 hours and 256 and $128 \mu \mathrm{g} / \mathrm{ml}$ after 48 hours), and C. glabrata was resistant to voriconazole (MIC90: $2 \mu \mathrm{g} / \mathrm{ml}$ after 24 hours and $4 \mu \mathrm{g} / \mathrm{ml}$ after 48 hours). All the yeasts tested were susceptible to amphotericin B, anidulafungin, micafungin and caspofungin.

\section{Discussion}

The aim of this study was to find an alternative for antifugal drugs currently used in the treatment of fungal infections. Since it has been demonstrated that myrtle essential oil has an antibacterial activity towards Escherichia coli, Staphylococcus aureus, Helicobacter pylori and other Gram-negative bacteria, we decided to test its antifungal activity against different species of Candida. The results obtained show good activity of the extract against $C$. albicans and $C$. tropicalis after 24 to 48 hours, and against $C$. parapsilosis after 24 hours. Good antifungal activity was also seen after 48 hours against $C$. glabrata, $C$. krusei and C. parapsilosis, but with higher MIC. At first sight, the results obtained seem higher than those obtained with the antimycotic tested, but lower than those reported in literature [17]. In particular, Mahboubi and colleagues obtained MIC values regarding $C$. albicans three times higher than those we registered [17]; however, Mahboubi's group studied the essential oil of Turkish myrtle, which differs from Italian myrtle essential oil. The essential oil of Turkish myrtle oil presents a higher percentage of linalool and linalyl acetate compared with Italian myrtle oil, the main components of which are $\alpha$ pinene and 1.8 cineole. Therefore, it is possible to conjecture that the different activity of myrtle essential oil is due to their different percentage compositions. Our work included not only $C$. albicans, but also $C$. non albicans strains, 
with promising results. These data are very important considering that some yeasts, such as $C$. glabrata and C. krusei, are resistant to conventional antifungal drugs, making them difficult to treat. Further studies are necessary to evaluate myrtle essential oil's exact chemical composition and toxicity.

\section{References}

1. Alem G, Mekonnen Y, Tiruneh M, Mulu A (2008) In vitro antibacterial activity of crude preparation of myrtle (Myrtus communis) on common human pathogens. Ethiopian Medical Journal 46: 63-69.

2. Clark AM (1996) Natural products as a resource for new drugs. Pharmaceutical Research 13: 1133-1144.

3. Romani A, Coinu R, Carta S, Pinelli P, Galardi C, Vincieri FF, Franconi F (2004) Evaluation of antioxidant effect of different extracts of Myrtus communis L. Free Radical Research 38: 97-103.

4. Deriu A, Branca G, Molicotti P, Pintore G, Chessa M, Tirillini B, Paglietti B, Mura A, Sechi LA, Fadda G, Zanetti S (2007) In vitro activity of essential oil of Myrtus communis $L$. against Helicobacter pylori. Int J Antimicrob Agents 30: 562563.

5. Zanetti S, Cannas S, Molicotti P, Bua A, Cubeddu M, Porcedda S, Marongiu B, Sechi LA (2010) Evaluation of the antimicrobial properties of the essential oil of Myrtus communis L. against clinical strains of Mycobacterium spp. Interdiscip Perspect Infect Dis Article ID 931530, 3 pages.

6. Douglas LJ (2003) Candida biofilms and their role in infection. Trends Microbiol 11: 30-36.

7. Dóczi I, Pető Z, Fodor E, Bereczki L, Nagy E, Hajdú E (2012) Evaluation of fungaemia infections in a Hungarian university hospital between 1996 and 2009. Acta Microbiol Immunol Hung 59: 29-41.

8. Khan ZU, Chandy R, Metwali KE (2003) Candida albicans strain carriage in patients and nursing staff of an intensive care unit: a study of morphotypes and resistotypes. Mycoses 46: 476-486.

9. Klepser ME (2001) Antifungal resistance among Candida species. Pharmacothery 21: 124S-132S.

10. Mustafa NK, Tanira MOM, Dar FK, Nsanze H (1999) Antimicrobial activity of Acacia nilotica sub spp, Nilotica fruit extracts. Pharm Pharmacol Communicat 5: 583-586.
11. Runyoro DKB, Matee MIN, Ngassapa OD, Joseph CC, Mbwambo ZH (2006) Screening of Tanzanian medicinal plants for anti-Candida activity. BMC Com Alternat Med 30: 6-11.

12. Khan R, Zakir M, Afaq SH, Latif A, Khan AU (2010) Activity of solvent extracts of Prosopis spicigera, Zingiber officinale and Trachyspermum ammi against multidrug resistant bacterial and fungal strains. J Infect Dev Ctries 4: 292-300.

13. Bakkali F, Averbeck S, Averbeck D, Idaomar M, (2008) Biological effects of essential oils - a review. Food and Chemical Toxicology 46: 446-475.

14. Palmeira de Oliveira R, Viegas DA, Amaral MH, Palmeira de Oliveira A, Salgueiro L, Cavaleiro C, Breitenfeld L, Martinez-de-Oliveira J (2011) Formulac, ões com óleos essenciais: Desenvolvimento de um lápis medicamentoso de Helichrysium italicum. Revista de Fitoterapia 11: 57-72.

15. Terlecka JA, Philipp A, Cros du Morrisey CO, Spelman D (2006) Rapid differentiation of $C$. albicans from non $C$. albicans species by germe tube test directly from Bact alert blood culture bottles. Mycoses 50: 48-51.

16. Clinical and Laboratory Standards Institute (2008) Reference method for broth diluition antifungal susceptibility testing of yeasts: approved standard, 3rd ed., M27-A3. Clinical and Laboratory Standards Institute, Wayne, PA.

17. Mahboubi $\mathrm{M}$, and Ghazian Bidgoli $\mathrm{F}$ (2010) In vitro sinergistic efficacy of combination of amphotericin B with Myrtus communis essential oil against clinical isolates of Candida albicans. Phitomedicine 17: 771-774.

\section{Corresponding author}

Sara Cannas

Dipartimento di Scienze Biomediche

Microbiologia Sperimentale e Clinica

Università degli Studi di Sassari

Via San Pietro 43b

07100 Sassari, Italy

Telephone/Fax: +39079229807/+39079212345

Email: sarac1977@libero.it

Conflict of interests: No conflict of interests is declared. 